\title{
Demanda por serviços públicos nos municípios do Espírito Santo: uma abordagem empírica
}

Edson Zambon Monte ${ }^{1}$

\begin{abstract}
Resumo: Este trabalho tem como objetivo estimar a demanda por bens públicos municipais no Estado do Espírito Santo, baseando-se na teoria do eleitor mediano. Os dados censitários referem-se ao ano de 2000. Verificou-se que as principais variáveis que explicam as despesas públicas municipais foram significativas e tiveram os sinais esperados, a saber: preço (tax-price), renda e população. A elasticidadepreço mostrou-se inelástica e a elasticidade-renda estimada permite referenciar que os bens públicos locais são essenciais (ou normais). O coeficiente do efeito congestionamento apresentou valor inferior à unidade, o que demonstra a presença de economias de escala a serem exploradas para os bens públicos.
\end{abstract}

Palavras-chave: Espírito Santo; teoria do eleitor mediano; regressão múltipla.

\section{Demand for local public services of Espírito Santo: an empirical approach}

\begin{abstract}
The object of this paper is to estimate the demand for local public services of Espírito Santo, based on the median voter theory and the traditional econometric approach. Census data refer to 200o. It was found that the main variables that explain municipal costs were significant and had the expected signs, namely: price (tax-price), income and population. The price elasticity was found to be inelastic and the estimated income elasticity allows reference to the local public goods are essential (or normal). The coefficient of the congestion effect showed a value less than unity, demonstrating the presence of economies of scale to be exploited for public goods.
\end{abstract}

Key-words: Espírito Santo; the median voter theory; multiple regression.

JEL: $\mathrm{C}_{31} ; \mathrm{H}_{70} ; \mathrm{H}_{72}$.

1 Mestre em Economia. Professor da Universidade Federal do Espírito Santo (UFES).

E-mail: edsonzambon@yahoo.com.br 


\section{Introdução}

As bases para o estudo das despesas ${ }^{2}$ públicas foram lançadas por Samuelson (1954). Tibeout (1956) trouxe avanços teóricos em relação aos gastos públicos abordando as particularidades dos mesmos a nível local. No entanto, até o início dos anos de 1970 as pesquisas empíricas relacionadas às despesas públicas baseavam-se em abordagens ad hoc (Bergstrom e Goodman, 1973). Conforme Barcelos (2007), no período, embora os modelos propostos contassem com inúmeras variáveis para analisar os gastos públicos, havia muita dificuldade em explicar a escolha destas variáveis.

A partir, então, da década de 1970, diversos estudos foram realizados com o intuito de verificar os determinantes das despesas públicas locais, tendo como referência os pressupostos microeconômicos da teoria do consumidor. Uma característica em comum, dentre várias destas pesquisas, é a utilização da teoria do eleitor mediano. Este método busca, por meio da agregação das demandas individuais, obter a demanda comunitária, ou seja, as decisões públicas locais são determinadas pela escolha do eleitor mediano.

Mesmo sendo bastante difundida, a teoria do eleitor mediano tem recebido algumas críticas. Uma delas refere-se à agregação de preferências individuais para representar uma comunidade como um todo. No mais, conforme apresentado por Mendes (2005), outros problemas são: erros de medida e presença de heterocedasticidade entre as variáveis, o que pode comprometer os resultados. Também existem questionamentos quanto às variáveis do modelo que não são observadas diretamente, como o tax-price ${ }^{3}$, utilizando-se proxies para representá-las. Outra questão é que as elasticidades estimadas não medem a demanda por serviços públicos, mas as despesas públicas.

Em contraponto a estas críticas, estudos vêm demonstrando a robustez do modelo do eleitor mediano, sendo este considerado válido no que se refere ao comportamento do eleitor mediano determinar o comportamento fiscal do governo. Mendes (2005) faz referência a alguns destes autores: Borcherding e Deacon (1972), Bergstrom e Goodman (1973), Pommerehne (1978), Edwards (1990), Turnbull e Djoundourian (1994), Turnbull e Chang (1998) e Aronsson et al. (2000), Dahlberg e Johansson (2000) e Sanz e Velazquez (2002).

Utilizando a teoria do eleitor mediano, Mendes (2005) estimou uma curva de demanda dos serviços públicos dos municípios brasileiros, considerando, como variável dependente, dentre outras, a despesa global. Os resultados encontrados pelo autor demonstram, por exemplo, que as variáveis preço (tax-price), renda e população foram significativas e com sinal de acordo com a teoria econômica. No caso de bens como educação e saúde, estes foram vistos como meritórios ou de "luxo", pois a elasticidade encontrada foi maior que a unidade. Resultados semelhantes foram encontrados por Mendes e Souza (2006), considerando como variável dependente a despesa global per capita.

2 Nesta pesquisa despesas e gastos são sinônimos.

3 Custo individual de se adquirir uma unidade adicional de serviço público local. 
Barcelos (2007) analisou a demanda por bens públicos locais a partir da despesa orçamentária total e setorial dos municípios brasileiros. Após a análise empírica, verificou-se que as variáveis renda, preço-fiscal, população total, intensidade da pobreza, dentre outras, são relevantes para explicar as despesas públicas dos municípios brasileiros.

De acordo com Stiglitz (apud Giambiagi e Além, 2000, p. 53), "o gasto do governo afeta uma gama diversa de atividades, algumas das quais, em algum momento, provavelmente teve, tem ou terá algo a ver com a vida de cada um de nós". Vale ressaltar que as despesas públicas não afetam a população somente pelo lado da própria despesa, ou seja, pela oferta de serviços públicos, mas também pelo lado do financiamento a estes gastos, que em geral são financiados pela cobrança de tributos dos diversos atores da sociedade. $\mathrm{Ou}$ seja, uma pessoa é, ao mesmo tempo, contribuinte e beneficiário dos gastos públicos.

Conforme Rezende (2001), o demanda por um bem público envolve duas reações de sentidos opostos. A primeira refere-se à satisfação advinda do próprio consumo, que depende da utilidade atribuída pelo indivíduo ao bem considerado. A segunda diz respeito ao pagamento de tributos (visto como sacrifício ou desutilidade) para financiar a produção de bens públicos. Este sacrifício reduz a renda disponível para o consumo de bens privados.

Segundo Giambiagi e Além (2000) existem funções que são eminentemente do governo, sendo que algumas delas também são realizadas pelo setor privado. Dentre as mais importantes estão: a) saúde; b) educação; c) defesa nacional; d) policiamento; e) regulação; f) justiça; e, g) assistencialismo. Estas funções impactam diretamente nas despesas do setor público. Ainda, segundo os autores, os gastos públicos elevaram-se fortemente ao longo do século XX, devido, principalmente, aos seguintes fatores: i) fatores demográficos associados ao envelhecimento progressivo da população; ii) urbanização; iii) crescimento do Produto Interno Bruto (PIB) per capita; e, iv) aumento do preço relativo dos serviços.

Levando-se em consideração que os gastos públicos vêm aumentando nas últimas décadas, e que os indivíduos são parte "financiadora" destes gastos, ganha cada vez mais ênfase os olhares para o controle destas despesas, seja em função de como financiá-las, de como realizá-las ou em como priorizar a destinação (aplicação) das mesmas. Neste particular, é cada vez mais relevante a realização de estudos que indiquem os fatores que impactam nos gastos públicos, em particular, nos de nível municipal. Dessa forma, este trabalho tem como objetivo estimar a demanda por bens públicos locais no Estado do Espírito Santo, baseando-se na teoria do eleitor mediano e empregando as técnicas de regressão tradicionais. 


\section{Modelo do eleitor mediano ${ }^{4}$}

No modelo do eleitor mediano os indivíduos maximizam uma função de utilidade, escolhendo entre dois bens, $x$ (privado) e $z$ (público), e sujeitos a uma restrição orçamentária. Assume-se que o preço do bem privado é igual a 1 (um), e que todos as pessoas dentro de uma mesma localidade consomem a mesma quantidade do bem público (preço $p_{z}$ ).

Assim, o leitor mediano exerce seu poder de voto buscando maximizar a função de utilidade expressa na Equação 1:

$U=U\left(x_{i}, z\right)$

sendo que está maximização está sujeita a uma restrição orçamentária (Equação 2):

$y_{m}=x+t_{i} b_{m}$

em que $y_{m}$ é a renda do eleitor mediano; $b_{m}$, base do imposto; e, $t_{i}$, parcela do imposto.

No caso das demandas individuais, estas também dependem da restrição orçamentária do governo, conforme Equação 3:

$\not=G+B$,

em que $c$ é o custo médio ou marginal constante da produção do serviço público; $\mathbb{B}$, receitas totais dos impostos; e, $G$, transferências intergovernamentais recebidas pela comunidade. Resolvendo a Equação 3 para $t$, chega-se a Equação 4:

$t=\frac{[Z-G] .}{B}$

Bocherding e Deacon (1972) demonstraram que, devido ao congestionamento (rivalidade), o consumo do bem público depende não somente do seu volume total $(Z)$, mas também do tamanho da população de determinada comunidade $(N)$. Dessa maneira, baseando-se nos autores, descreve-se a função congestionamento, conforme Equação 5:

$$
Z=N^{\gamma} z
$$

em que $\gamma$ representa o efeito congestionamento ou rivalidade. Este parâmetro também pode ser considerado uma medida de publicização do bem, já que reflete sua maior característica; a não-rivalidade. 
Caso $\gamma$ seja igual a unidade, o bem é tipicamente privado, não havendo benefícios de economias de escala para sociedade e, sendo o consumo individual, igual a $\frac{1}{N}$. Quando $\gamma$ for igual a zero, o bem é público puro (o bem é indivisível e não rival). Se $\gamma$ for maior que um o bem é considerado supercongestionado marginalmente. Por fim, em situações em que $\gamma$ está entre zero e um, o bem é considerado semipúblico (ou semiprivado), com certos padrões de congestionamento, mas com economias de escala a serem exploradas.

Tomando-se as Equações 4 e 5, e substituindo-as na Equação 2 (restrição orçamentária do eleitor mediano), encontra-se a Equação 6:

$$
y_{a}=y_{m}+g\left(\frac{b_{m}}{b}\right)=x+\left(\frac{b_{m}}{b}\right) \mathrm{N}^{\gamma-1} z
$$

em que $y_{a}$ é a receita média aumentada por uma parcela das transferências intergovernamentais per capita; $g=\frac{G}{N}$; e, $b=\frac{B}{N}$, sendo $B$ a base do imposto total local. A renda total do eleitor mediano deve cobrir suas despesas privadas e sua parcela de custo na aquisição do serviço público $\left(\frac{b_{m}}{b}\right) \mathbb{N}^{\gamma-1} z$ A Equação 6 pode ser reescrita, dando origem a Equação 7:

$$
x=y_{m}+\left(\frac{b_{m}}{b}\right)\left[g-\mathbb{N}^{\gamma-1} z\right]
$$

Ao inserir a Equação 7 na Equação 1, chega-se a Equação 8:

$$
\max U=U\left[y_{m}+\left(\frac{b_{m}}{b}\right)\left[g-\mathbb{N}^{\gamma-1} z\right], z\right] \text {. }
$$

Agora, supondo-se que a maximização da Equação 8 leva a função de demanda do eleitor mediano, para um serviço público local $(z)$, obtem-se a Equação 9:

$$
z=z\left[y_{a}, \frac{b_{m}}{b}, N\right] \text {. }
$$

Considerando-se o preço do serviço público (tax-price) como o custo individual de se adquirir uma unidade adicional de serviço público local, ele pode ser derivado diferenciando-se $y_{a}$ com relação à $z$. Tem-se, então, o preço do imposto dado pela Equação 10: 


$$
\frac{\delta y_{a}}{\delta z}=p=\left(\frac{b_{m}}{b}\right) \mathrm{N}^{\gamma-1}
$$

O consumidor sabe seu próprio custo ou preço do imposto e é capaz de determinar a quantidade de serviço ${ }^{5}$ para a comunidade. Ao supor que a função de demanda da Equação $9\left[z=f\left(p_{i}, y_{a}\right]\right.$ possui elasticidades de renda e de preço constantes, determina-se a Equação 11:

$z=\alpha p^{\beta_{1}} y_{a}^{\beta_{2}}$.

Substituindo a Equação 10 na Equação 11, a demanda de $z$ fica expressa pela Equação 12:

$z=\alpha\left[\left(\frac{b_{m}}{b}\right) \mathcal{N}^{\gamma-1}\right]^{\beta_{1}} y_{a}^{\beta_{2}} \cdot$

Inserindo-se a Equação 12 na Equação 5, encontra-se a Equação 13:

$Z=\mathbb{N}^{\gamma}=\alpha\left[\left(\frac{b_{m}}{b}\right) \mathbb{N}^{\gamma-1}\right]^{\beta_{1}} y_{a}^{\beta_{2}} N^{\gamma}$.

Por fim, incluindo um vetor $\Omega$ de características sócio-econômicas, que se acredita, afetam a demanda por bens públicos, e multiplicando a Equação 13 por $p$, tem-se uma função estimável da despesa pública municipal $(E)$ dada pela Equação 14:

$$
E=\not 2=p z N^{\gamma}=\alpha\left[\left(\frac{b_{m}}{b}\right) N^{\gamma-1}\right]^{\beta_{1}} y_{a}^{\beta_{2}} N^{\gamma} \Omega^{\beta_{i}} \cdot
$$

Aplicando a propriedade logarítmica, a equação para analisar as despesas públicas municipais fica expressa por:

h $E=k+\beta_{1}\left[\mathrm{~h}\left(\frac{b_{m}}{b}\right)\right]+\beta_{2} \mathrm{~h}\left(y_{a}\right)+\beta_{3} \mathrm{~h}(N)+\sum_{i=4}^{n} \beta_{i}\left(\mathrm{~h} \Omega_{i}\right)+\varepsilon_{i}$

em que $k=\mathrm{h} \alpha+\beta_{1} \mathrm{~h} c ; \beta_{1}$, elasticidade-preço da demanda; e, $\beta_{3}$ (elasticidade-população), satisfaz a Equação 16:

$\beta_{3}=\gamma\left(1+\beta_{1}\right)-\beta_{1}$.

5 "Existem três razões que podem explicar o porquê dos eleitores perceberem, as vezes, de maneira errada os custos dos serviços públicos: ilusão fiscal, efeito flypaper e, complexidade da receita.” (Reiter e Weichenrieder, 1997: 21). 


\section{Modelo analítico}

A análise de regressão é um dos pontos mais importantes da análise econométrica, onde se busca verificar, descrever e estimar relações entre dois tipos de variáveis, a saber: variável dependente (ou variável a ser explicada) e variável(eis) independente(s) ou explicativa(s).

Nesta pesquisa, com o intuito de explicar as despesas públicas dos municípios capixabas, será utilizada a análise de regressão linear múltipla, fazendo-se o uso do método de Mínimos Quadrados Ordinários (MQO).

O método de MQO é representado, de forma simplificada, pela Equação 17 (Gujarati, 2006):

$$
Y_{i}=\hat{\beta}_{1}+\hat{\beta}_{2} X_{2 i}+\hat{\beta}_{3} X_{3 i}+\ldots+\hat{\beta}_{k} X_{k}+\hat{u}_{i}, i=1,2,3, \ldots, n,
$$

em que $Y_{i}$ é a variável dependente; $X_{i}$, variáveis explicativas; $\beta_{1}$, intercepto; $\beta_{2} \ldots \beta_{k}$, coeficientes parciais de inclinação; $u_{i}$, termo de perturbação estocástico; e, $i$, i-ésima observação, sendo $n$ o tamanho de população.

A Equação 17 pode ser representada da seguinte forma:

$$
y=X \hat{\beta}+\hat{u},
$$

ou, ainda, na forma matricial dada pela Equação 19:

$$
\begin{aligned}
& \begin{array}{c}
{\left[\begin{array}{c}
Y_{1} \\
Y_{2} \\
\vdots \\
Y_{n}
\end{array}\right]=\left[\begin{array}{ccccc}
1 & X_{2} & X_{3} & \cdots & X_{k 1} \\
1 & X_{2} & X_{3} & \cdots & X_{k 2} \\
\vdots & \vdots & \vdots & \vdots & \vdots \\
1 & X_{2 n} & X_{3 n} & \cdots & X_{k}
\end{array}\right]\left[\begin{array}{c}
\hat{\beta}_{1} \\
\hat{\beta}_{2} \\
\vdots \\
\hat{\beta}_{k}
\end{array}\right]+\left[\begin{array}{c}
\hat{u}_{1} \\
\hat{u}_{2} \\
\vdots \\
\hat{u}_{k}
\end{array}\right],} \\
y=\frac{\hat{\beta}}{+}
\end{array} \\
& n \times 1 \quad n \times k \quad k \times 1 \quad n \times 1 \text {, }
\end{aligned}
$$

em que $y$ é o vetor coluna $n \times 1$ de observações sobre a variável dependente $Y ; X$, matriz $n \times k$, dando $n$ observações sobre $k-1$ variáveis $X_{2}$ e $X_{k}$ sendo que a primeira coluna de $1 s$ representa o termo de intercepto. Esta matriz também é conhecida como matriz de dados; $\beta$, vetor coluna $k \times 1$ dos parâmetros desconhecidos $\beta_{1}, \beta_{2}, \ldots, \beta_{k} ; \mathrm{e}, u$, vetor coluna $n \times 1$ das $n$ perturbações $u_{i}$. 


\section{Variáveis e dados}

Os dados deste estudo são do tipo corte transversal (cross-section), sendo, em sua maioria, de ordem censitária, o que limita a disponibilidade de dados mais recentes. Por isto, o período de análise é o exercício de 2000, ano em que ocorreu o penúltimo censo realizado pelo Instituto Brasileiro de Geografia e Estatística (IBGE), e que permite extrair todas as variáveis necessárias para a análise. Grande parte dos dados foi obtida do IBGE, com exceção da receita e da despesa local, provenientes da Secretaria do Tesouro Nacional (STN), e da intensidade da pobreza, obtida do Programa das Nações Unidas para o Desenvolvimento (PNUD). As variáveis estão descritas na Tabela 1.

TABELA 1. VARIÁVEIS UTILIZADAS

\begin{tabular}{cl}
\hline \multicolumn{1}{c}{ Variável dependente } & \multicolumn{1}{c}{ Descrição } \\
\hline Despesa global $(E)$ & $\begin{array}{l}E: \text { despesa orçamentária total de cada } \\
\text { município. }\end{array}$ \\
\hline
\end{tabular}

Variáveis explicativas essenciais

Descrição

Parcela do imposto (TAXPRICE)

Razão entre a receita tributária per-capita local e o total de receita orçamentária de cada município. Uma medida de esforço fiscal.

Soma entre a renda média de cada município e produto da multiplicação do

Renda média ampliada (RENDA) tax-price pelo montante das transferências intergovernamentais recebidas por cada município.

População do município (POPULACAO) Número de habitantes de cada município.

\section{Variáveis explicativas de contexto \\ [matriz $\Omega_{]}$ \\ características sócio-econômicas, demográficas e geográficas}

Desenvolvimento humano (IDH)

Esperança de vida (ESPERANCAVIDA)

Taxa de alfabetização (TXALFABET)

Intensidade da pobreza

(INTENSIDADEPOBREZA)

Percentual da população com até 15 anos (POPATE15ANOS)

\section{Descrição}

Índice de desenvolvimento humano de cada município.

Número médio de anos que as pessoas vivem após seu nascimento. Como uma proxy para os serviços de saúde.

Razão entre a população acima de 15 anos alfabetizada e a população total do município.

Distância que separa a renda domiciliar per capita média dos indivíduos pobres (ou seja, dos indivíduos com renda domiciliar per capita inferior à linha de pobreza de $\mathrm{R} \$ 75,50)$ do valor da linha de pobreza ( $\mathrm{R} \$ 37,50)$, medida em termos de percentual do valor dessa linha de pobreza.

Razão entre a população de até 15 anos e a população total do município. 


\section{TABELA 1 (continuação). VARIÁVEIS UTILIZADAS}

Percentual da população com mais de 65 anos (POPMAISDE65ANOS)

Percentual da população rural (POPRURAL)

Percentual da população urbana (POPURBANA)

Distância da capital (DISTCAPITAL)

Densidade demográfica (DENSDEMOG)

Percentual da renda proveniente das transferências intergovernamentais (TRANSINTERGOV)
Razão entre a população com mais de 65 anos e a população total do município.

Informação censitária obtida pela razão entre a população residente na área rural e a população total.

Informação censitária obtida pela razão entre a população residente na área urbana e a população total.

Distância, em linha reta, do município à capital do Estado, em Km.

Razão entre a população residente total e a área do município (hab. $/ \mathrm{km}^{2}$ ).

Participação percentual das rendas provenientes das transferências governamentais na renda total do município.

Fonte: elaborado pelo autor, tomando como base Barcelos (2007).

O Estado do Espírito Santo possui 78 municípios. No entanto, este estudo abordará somente 76 municípios, uma vez que Governador Lindemberg foi criado em 2001 e Bom Jesus do Norte não possui alguns dados disponíveis.

\section{Resultados e discussões}

Na Tabela 2 são apresentados os resultados referentes às estimativas de MQO para as despesas públicas municipais capixabas (modelo completo). Ressalta-se aqui, que foram realizados todos os testes estatísticos a fim de verificar a boa especificação do modelo e possíveis problemas com as hipóteses do Modelo de Regressão Linear Clássico (MRLC), como: teste de normalidade (Jarque-Bera); teste de especificação do modelo (RESET); teste de autocorrelação; e, teste de heterocedasticidade de White. Este último revelou a rejeição da hipótese de homocedascidade. Logo, o modelo foi corrigido pelo método de White.

Verifica-se que as variáveis expectativa de vida, intensidade da pobreza, população de até 15 anos e população com mais de 65 anos, não foram significativas, do ponto de vista estatístico, na determinação das despesas públicas dos municípios do Espírito Santo.

Além disso, as variáveis taxa de alfabetização, intensidade da pobreza, distância da capital e transferências intergovernamentais apresentaram os sinais de seus coeficientes contraditórios aos esperados. Para as variáveis intensidade da pobreza, distância da capital e transferências intergovernamentais esperava-se encontrar sinais positivos. Para a variável alfabetização esperava-se encontrar sinal negativo. 
A estatística $F$ demonstrou que a regressão global é estatisticamente significativa e o coeficiente de determinação $\left(R^{2}=0,9631\right)$ revelou que $96,31 \%$ das variações das despesas públicas locais são explicadas pelas variações das variáveis explicativas presentes no modelo.

A elasticidade-preço negativa indica que a medida que o esforço fiscal aumenta (eleva-se a carga tributária municipal), reduz-se a demanda por serviços públicos locais. Neste particular, uma elevação de $1 \%$ no preço do bem público (tax-price) acarreta em uma redução de 0,2048\% na demanda por bens públicos. O resultado sugere uma demanda inelástica (bens básicos ou essenciais), sendo que o coeficiente é superior ao encontrado por Mendes (2005) e Barcelos (2007), quando estimaram a demanda por bens públicos municipais no Brasil, utilizando como variável dependente a despesa global. Sendo a demanda inelástica, possíveis políticas locais que elevem o preço do bem público tendem a impactar pouco na demanda pelos mesmos, o que de certa forma daria uma boa margem para possíveis aumentos de impostos pelo governo.

TABELA 2. DETERMINANTES DA DESPESA LOCAL: MODELO COMPLETO

\begin{tabular}{lcccc}
\hline \multicolumn{1}{c}{ Variáveis } & Coeficientes & Erro-padrão & Valor $\boldsymbol{p}$ & $\boldsymbol{p}$-valor \\
\hline C & $-8,2217^{\mathrm{ns}}$ & 9,4015 & $-0,8745$ & 0,3853 \\
L (TAXPRICE) & $-0,2048^{* * *}$ & 0,0628 & $-3,2582$ & 0,0018 \\
L (RENDA) & $0,5580^{* *}$ & 0,2678 & 2,0840 & 0,0414 \\
L (POPULACAO) & $0,6888^{* * *}$ & 0,0728 & 9,4605 & 0,0000 \\
L (IDH) & $-3,5664^{*}$ & 2,0236 & $-1,7625$ & 0,0965 \\
L (ESPERANÇAVIDA) & $1,2481^{\mathrm{ns}}$ & 1,1291 & 1,1055 & 0,2733 \\
L (TXALFABET) & $2,0845^{* *}$ & 0,9459 & 2,2038 & 0,0313 \\
L (INTENSIDADEPOBREZA) & $-0,1270^{\mathrm{ns}}$ & 0,2697 & $-0,4710$ & 0,6393 \\
L (POPATE15ANOS) & $0,0568^{\mathrm{ns}}$ & 0,1732 & 0,3279 & 0,7441 \\
L (POPMAISDE65ANOS) & $0,0622^{\mathrm{ns}}$ & 0,1492 & 0,4173 & 0,6779 \\
L (POPRURAL) & $-0,0628^{* * *}$ & 0,0173 & $-3,6243$ & 0,0006 \\
L (POPURBANA) & $0,2619^{* * *}$ & 0,0939 & 2,7893 & 0,0070 \\
L (DISTCAPITAL) & $-0,2196^{* * * *}$ & 0,0518 & $-4,2384$ & 0,0001 \\
L (DENSDEMOG) & $-0,2976^{* * *}$ & 0,0480 & $-6,2047$ & 0,0000 \\
L (TRANSINTERGOV) & $-0,5431^{* * *}$ & 0,2640 & $-2,0574$ & 0,0439 \\
PAR, CONGESTIONAMENTO & & & 0,6087 & \\
\hline
\end{tabular}

Fonte: elaborado pelo autor a partir dos dados da pesquisa.

Nota: 1$) * *$ Significativo a $1 \%,{ }^{* *}$ Significativo a $5 \%,{ }^{*}$ Significativo a $10 \%$, ns Não-significativo a $10 \%$; 2) $\mathrm{L}=$ Indica que as variáveis estão expressas em logaritmos. 
No caso da elasticidade-renda, esta é positiva e inferior à unidade, demonstrando que os bens públicos são de caráter normal ou essencial para a população dos municípios do Espírito Santo, embora não possam ser caracterizados como bens superiores. O aumento de $1 \%$ na renda provoca um crescimento de $0,5580 \%$ na demanda por bens públicos locais.

Pelas características encontradas para a elasticidade-preço da demanda e para a elasticidade-renda, e baseando na teoria microeconômica, nota-se que, em média, os bens públicos locais são considerados de primeira necessidade para a população dos municípios capixabas, demonstrando a importância de políticas públicas bem elaboradas para estas localidades.

Assim como esperado, o coeficiente da variável população total indica que populações maiores representam maiores despesas públicas. $\mathrm{O}$ aumento da população por si só eleva os gastos públicos, uma vez que isto elevará a demanda por bens oferecidos pelo governo, como: educação, saúde, saneamento, dentre outros.

Destaca-se aqui, o elevado coeficiente (negativo) encontrado par a variável IDH. Elevando-se o IDH em 1\% reduz-se as despesas públicas municipais em 3,5664\%. Isto representa que, à medida que os municípios capixabas se desenvolvem, ocorre uma forte queda da demanda das comunidades por bens públicos. Este resultado é expressivo, mas deve-se levar em conta que o IDH abrange três indicadores fundamentais: renda, longevidade e educação, tornando uma variável essencial para redução das despesas públicas.

O coeficiente estimado para a população rural foi negativo ao passo que o coeficiente estimado para a população urbana foi positivo. Isto implica que, o indivíduo localizado nos centros urbanos demanda mais gastos públicos que os localizados em comunidades rurais (neste caso reduz-se a demanda por bens públicos). Em suma, os centros urbanos tendem à ter uma infraestrutura muito maior que o meio rural. A especialização e a diversidade de bens públicos oferecidos nos centros urbanos é muito maior do que nas comunidades rurais, sendo que em certas localidades a população rural desloca-se para os centros urbanos em busca dos serviços públicos oferecidos nestes centros.

A variável densidade demográfica mostrou-se significativa e com sinal negativo. Isto pode estar associado aos retornos de escala crescentes presentes em pequenos municípios. Os municípios com menor população possuem custos médios dos serviços públicos mais elevados, limitando a exploração de economias de escala na produção dos serviços públicos, o que torna a aplicação de recursos ineficiente. Do contrário, a maior densidade demográfica reduz os custos dos serviços e permite a redução de despesas públicas (Mendes, 2005).

O coeficiente de congestionamento foi estimado conforme Equação 16. Este refere-se ao grau de publicização dos bens públicos municipais. O valor encontrado $(0,6087)$ está próximo daqueles encontrados por Mendes (2005) e 
Barcelos (2007) para os municípios brasileiros, evidenciando que, em média, existem economias de escala a serem exploradas pelos municípios do Espírito Santo. Dessa forma, os bens públicos dos municípios capixabas podem ser classificados como semipúblicos ou semiprivados. Resultado semelhante foi encontrado por MacMillan et al. (1981) ao estudar pequenas comunidades urbanas do Canadá. Já Borcherding e Deacon (1972), Bergstrom e Goodman (1973) encontraram estimativas próximas ou maiores que a unidade.

A fim de verificar a robustez do modelo, observa-se na Tabela 3 (modelo reduzido) as estimativas considerando somente as variáveis significativas apresentadas na Tabela 2. Assim, foram eliminadas da nova regressão as variáveis insignificantes estatisticamente e o termo relativo à constante $(C)$. Todas as variáveis do novo modelo foram significativas, e o coeficiente de determinação obtido foi igual a 0,9617, apresentando uma pequena redução em relação ao modelo completo.

\section{TABELA 3. DETERMINANTES DA DESPESA LOCAL: MODELO REDUZIDO}

\begin{tabular}{lcccc}
\hline \multicolumn{1}{c}{ Variáveis } & Coeficientes & Erro-padrão & Valor $\boldsymbol{p}$ & $\boldsymbol{p}$-valor \\
\hline L (TAXPRICE) & $-0,1818^{* * * *}$ & 0,0578 & $-3,1432$ & 0,0025 \\
L (RENDA) & $0,5065^{* * *}$ & 0,2166 & 2,3379 & 0,0224 \\
L (POPULACAO) & $0,6847^{* * *}$ & 0,0674 & 10,1648 & 0,0000 \\
L (IDH) & $-1,8622^{* *}$ & 1,0340 & $-1,8009$ & 0,0763 \\
L (TXALFABET) & $1,6248^{* * *}$ & 0,3500 & 4,6424 & 0,0000 \\
L (POPRURAL) & $-0,0544^{* * *}$ & 0,0176 & $-3,0866$ & 0,0030 \\
L (POPURBANA) & $0,2444^{* * *}$ & 0,0836 & 2,9224 & 0,0048 \\
L (DISTCAPITAL) & $-0,2941^{* * *}$ & 0,0508 & $-5,7877$ & 0,0000 \\
L (DENSDEMOG) & $-0,2278^{* * *}$ & 0,0626 & $-3,6392$ & 0,0005 \\
L (TRANSINTERGOV) & $-0,5105^{* *}$ & 0,2510 & $-2,0344$ & 0,0459 \\
PAR, CONGESTIONAMENTO & & 0,7071 & & \\
\hline
\end{tabular}

Fonte: elaborado pelo autor a partir dos dados da pesquisa.

Nota: 1$) * *$ Significativo a $1 \%,{ }^{* *}$ Significativo a $5 \%,{ }^{*}$ Significativo a $\left.10 \% ; 2\right) \mathrm{L}=$ Indica que as variáveis estão expressas em logaritmos; 3) Foram realizados todos os testes estatísticos a fim de verificar a boa especificação do modelo e possíveis problemas com as hipóteses do MRLC. O modelo foi estimado considerando a correção de White. 
Ainda, conforme Tabela 3, observa-se que as variáveis taxa de alfabetização, distância da capital e transferências intergovernamentais permanecem com sinais contrários aos esperados pela teoria econômica. A variável com maior efeito sobre as despesas públicas continua sendo o IDH.

No mais, pela elasticidade-preço e pela elasticidade-renda do modelo reduzido, verifica-se novamente que os bens públicos são de primeira necessidade para os municípios do Espírito Santo. A demanda pelos mesmos é inelástica e os bens são normais ou essenciais.

Por fim, destaca-se que o parâmetro de congestionamento encontrado no modelo reduzido $(0,7071)$ é um pouco maior que o coeficiente estimado para o modelo completo $(0,6087)$. No entanto, mesmo com está pequena divergência, nos dois casos o parâmetro demonstra que os bens públicos nos municípios capixabas são semipúblicos (ou semiprivados), apresentando economias de escala a serem exploradas.

\section{Conclusão}

O objetivo deste estudo foi de estimar a demanda por serviços públicos municipais no Estado do Espírito Santo, buscando verificar como as despesas públicas locais do estado variam de acordo com fatores sócio-econômicos, demográficos e geográficos. O trabalho baseou-se no modelo do eleitor mediano.

Verificou-se que as principais variáveis que explicam as despesas públicas municipais foram significativas e tiveram os sinais esperados, a saber: preço (tax-price), renda e população. Vale mencionar o forte impacto que a variável IDH tem sobre as despesas públicas locais.

De acordo com os resultados, os bens públicos são de primeira necessidade para as comunidades dos municípios capixabas. Despesas mal planejadas tendem a comprometer em muito o bem-estar das pessoas destas localidades. Políticas que elevem o IDH, por exemplo, além de melhorarem a situação geral da população, tende a reduzir de forma relevante os gastos públicos locais. Em localidades mais desenvolvidas, a população tende a depender menos dos gastos governamentais.

Nota-se ainda, que o coeficiente do efeito congestionamento apresentou valor inferior à unidade, o que demonstra a presença de economias de escala a serem exploradas para os bens públicos. Isto indica que o consumo dos bens públicos locais no Espírito Santo não está congestionado, e que novos consumidores podem demandá-los, repartindo os custos do fornecimento, sem perda de utilidade. Dessa forma, verifica-se que ainda há espaço para provisão de bens públicos por parte das autoridades dos municípios do Espírito Santo, especialmente no que se refere aos bens que são obrigações do setor público. 
Conforme já mencionado, existem funções que são eminentemente do governo: a) saúde; b) educação; c) defesa nacional; d) policiamento; e) regulação; f) justiça; e, g) assistencialismo (Giambiagi e Além, 2000). Estas impactam diretamente nas despesas do setor público, sendo que os indivíduos (população) são, ao mesmo tempo, demandantes e financiadores destes gastos públicos. Além disso, destaca-se que o aumento dos gastos dos indivíduos com bens públicos leva à redução do consumo de bens privados, dada a redução da renda disponível.

Neste contexto, o que se observa ao longo dos anos é que as despesas públicas têm aumentado significantemente, porém, na maioria das vezes, somente em termos quantitativos e, não, qualitativos. Sendo assim, este estudo, ao quantificar os determinantes das despesas públicas municipais do Espírito Santo, visa subsidiar as ações dos governos locais, de maneira que seus gastos sejam realizados da maneira mais eficiente possível, ou seja, buscando qualidade e não somente quantidade.

Em termos gerais, acredita-se que não basta somente a aumentar os gastos públicos, mesmo que seja nas funções essenciais do governo. O planejamento governamental adequado pode auxiliar em muito na tomada de decisões relativas à como financiar, realizar ou em como priorizar a destinação das despesas públicas. Gastar somente por gastar não é o ideal. Isto deve passar por uma abordagem mais ampla, de como gerir os gastos públicos. Torna-se, então, uma questão de gestão, que em muitos casos é fragilizada no setor público, e que o setor privado, em grande parte dos casos, faz de maneira eficiente. Assim, este estudo pretende, também, mesmo que pontualmente, contribuir para que as despesas públicas dos municípios do Espírito Santo sejam eficientes no conceito de gestão, realizando-se gastos onde realmente seja necessário, e com eficiência.

Por fim, considera-se que os resultados atingiram o objetivo proposto. No entanto, vale ressaltar que este ainda é um estudo inicial. Sugere-se que, para próximos estudos, seja analisada a possibilidade de segmentar os municípios capixabas por micro ou macro regiões, de forma a analisar se existem possíveis diferenças nos determinantes dos gastos públicos das mesmas ou, até mesmo, segmentar os municípios por número de habitantes. No mais, com a divulgação de novos dados censitários, propõe-se uma análise em dados em painel, com o intuito de verificar a evolução dinâmica das despesas públicas municipais capixabas. Esta análise em painel permitirá estudar, a partir desta pesquisa inicial, se o padrão de gastos públicos dos municípios capixabas permanece o mesmo ao longo do tempo. 


\section{Referências bibliográficas}

BARCELOS, C. L. K. (2007). Determinantes da despesa pública local: um estudo empírico dos municípios brasileiros a luz do teorema do eleitor mediano. [Dissertação de Mestrado]. Programa Multiinstitucional e Inter-regional de Pós-graduação em Ciências Contábeis (UnB, UFPA, UFPE e UFRN).

BERGSTROM, T. C. \& GOODMAN, R. P. (1973). "Private demands for public goods". American Economic Review, vol. 63 (3). URL [on-line]: <http://www.escholarship.org/uc/item/11j5d1t4>. Acesso em: $19 \mathrm{de}$ nov. de 2010.

BORCHERDING, T. E. \& DEACON, R. T. (1972). "The demand for the services of non-federal governments". The American Economic Review, vol. 62 (5), p. 891-901. URL [on-line]: <http://www.jstor.org/stable/ pdfplus/1815207.pdf $>$. Acesso em: 22 de nov. de 2010.

GIAMBIAGI, F. \& ALÉM, A. C. (2000). Finanças públicas: teoria e prática no Brasil. $2^{\text {a }}$ ed. Rio de Janeiro: Elsiever.

GUJARATI, D. N. (2006). Econometria básica. $4^{\mathrm{a}}$ ed. São Paulo: Elsevier.

MACMILLAN, M. L. \& WILSON, R. W. \& ARTHUR, L. M. (1981). "The publicness of local public goods: evidence from Ontario municipalities". Canadian Journal of Economics, vol. 14, (4), p. 596-608. URL [on-line]: <http://www.jstor.org/pss/134818>. Acesso em: 01 de dez. de 2010.

MENDES, C. C. (2005). A demanda por serviços públicos municipais no Brasil: a abordagem do eleitor mediano revisada. [Tese de Doutorado]. Programa de Pós-Graduação em Economia (UnB).

MENDES, C. C. \& SOUZA, M. C. S. (2006). "Estimando a demanda por serviços públicos nos municípios brasileiros". Revista Brasileira de Economia, vol. 60 (3), p. 281-296. URL [on-line]: <http://virtualbib.fgv.br/ojs/ index.php/rbe/article/view/941/508>. Acesso em: 23 de nov. de 2010.

REITER, M. \& WEICHENRIEDER, A. (1997). "Are public goods public? A critical survey of the demand estimates for local public services". Finanzarchiv, vol. 54 (3), p. 374-408.

REZENDE, F. A. (2001). Finanças públicas. $2^{\mathrm{a}}$ ed. São Paulo: Atlas, 382 p.

SAMUELSON, P. A. (1954). "The pure theory of public expenditure". The Review of Economics and Statistics, vol. 36 (4), p. 387-389.

TIEBOUT, C. M. (1956). "A pure theory of local expenditures". The Journal of Political Economy, vol. 64 (5), p. 416-424.

Recebido em: 18 de janeiro de 2011 Primeira resposta em: 28 de abril de 2011 Aceite em: 07 de junho de 2011 
MONTE, E. Z. Demanda por serviços públicos... 